\title{
ANTI-MÜLLERIAN HORMONE - A HORMONE WITH NARROW SPECTRUM OF ACTION AND BROAD SPECTRUM OF CLINICAL SIGNIFICANCE
}

\author{
Vilhelm Mladenov \\ UMHAT “Sveta Marina”, First Pediatric Clinic
}

\begin{abstract}
AMH (Anti-Müllerian Hormone, Müllerian Inhibiting Substance or Factor) is a member of the transforming growth factor - $\beta$ family, secreted by the fetal and prepubertal testicular Sertoli cells, and to a lesser amount by the granulosa cells of the small ovarian follicles. It plays an important role in the shaping of the male reproductive tract and is one of the baseline hormones in the diagnostics of the disorders of the sex development in the male. In the female, in the recent years AMH has become an important clinical tool for evaluation of the ovarian follicular reserve, for predicting ovarian response to gonadotropin stimulation in IVF treatment and for the diagnosis of hyperandrogenism. Recombinant AMH is a promising medication for the treatment of gynecological cancers and endometriosis.
\end{abstract}

\section{AMH - secretion and function}

AMH (Anti-Müllerian Hormone, Müllerian Inhibiting Substance or Factor) is a dimeric glycoprotein member of the transforming growth factor- $\beta$ (TGF- $\beta$ ) family. In the male it is produced by the testicular Sertoli cells and its expression is initiated by the end of the $7^{\text {th }}$ embryonic week. AMH causes regression of the Müllerian ducts (derivatives of the uterus, fallopian tubes and upper part of the vagina) and thus plays an important role in shaping the male reproductive tract (1). The initiation of AMH secretion is gonadotropin independent. Later AMH secretion is up regulated by $\mathrm{FSH}$ and down regulated by

\footnotetext{
Address for correspondence:

First Paediatric Clinic,

UMHAT „Sv. Marina“, First Paediatric Clinic,

1 „Hr.Smirnenski“ Blvd., Varna 9010, Bulgaria

e-mail:wilchelmm@yahoo.com
}

Received: June 12, 2014

Accepted: September 10, 2014 androgens. During fetal life and the first months after birth, when the levels of androgens are high, there is a physiological insensitivity of Sertoli cells to androgens because of lack of androgen receptor expression in the Sertoli cells nuclei (2). The levels of AMH in males are high from the end of the $7^{\text {th }}$ fetal week until the beginning of puberty, except for a transitional decline in the perinatal period. With the onset of puberty AMH production is inhibited by the increase of intratesticular testosterone, which overcomes the positive effect of FSH, and the onset of germ cell meiosis. Exogenous testosterone applications do not affect the AMH levels most probably because they do not lead to high intratesticular testosterone concentrations $(1,2)$.

In the female $\mathrm{AMH}$ is produced by the granulosa cells of the pre antral and small antral ovarian follicles and may act to modulate the early stages of follicular development by inhibiting the recruitment of further primordial follicles into the growing pool and reducing the sensitivity of antral follicles to FSH (3). AMH production ceases rapidly when follicular diameter reaches $8-10 \mathrm{~mm}(3,4)$. In a study per- 
formed in 804 healthy females at age ranging from infancy to the end of the reproductive period, Fong et al. found that AMH levels increase during childhood and reach a maximum at the age of 15.8 years, form a plateau until the age of 25 years and then start to decline yearly at a consistent rate until below the clinical detection limit by the age of 50 years or around 5 years before menopause (5). AMH levels in women are a reliable marker for the ovarian reserve of primordial follicles.

\section{$A M H$ - clinical use in the male}

In the male AMH is a useful marker for the presence of testicular tissue in such cases as suspected anorchia, bilateral cryptorchidism or more complex disorders of sex development (DSD) (6). Between $2^{\text {nd }}-6^{\text {th }}$ month after birth (the so called "minipuberty") the levels of gonadotropins and testosterone in boys are in the pubertal range but afterwards they normally decrease to undetectable values until the onset of puberty and cannot be used for evaluation of the hypothalamo-pituitary-gonadal axis. On the contrary, during that period the Sertoli cells remain active and the determination of AMH is very useful without the need of performing stimulation tests (1).

\section{6, XY DSD}

Disorders of sex development (DSD) are defined as congenital conditions in which development of chromosomal, gonadal and anatomical sex is atypical (7). Individuals with karyotype 46, XY have variable levels of genital ambiguity, ranging from undervirilized male to completely female external genitalia. In gonadal dysgenesis the fetal formation of the gonads is impaired. In the complete form the phenotype is female with normal external genitalia and preserved Müllerian structures while in the partial form the phenotype is variable, extending to normal male with infertility only. Several single gene disorders (SRY, SF-1, WT1, SOX9, etc.) or chromosomal rearrangements have been described as causes for gonadal dysgenesis but currently genetic diagnosis could be reached in only around $30 \%$ of the cases (8).

In accordance with the quite broad phenotypic spectrum of the gonadal dysgenesis the hormonal profiles show a variable reduction in AMH level. $\mathrm{AMH}$ is undetectable in anorchia and can be used to distinguish between that condition and bilateral cryptorchidism where it is in the normal range $(6,8)$. In conditions with impaired testosterone synthesis AMH is normal or high because of the lack of inhibitory effect of testosterone and the elevation of FSH, which up regulates AMH secretion (1). In androgen insensitivity syndromes (AIS), mutations in the androgen receptor gene cause complete lack of virilization (complete androgen insensitivity syndrome, CAIS) and feminization of the external genitalia or partial undervirilisation (partial androgen insensitivity syndrome, PAIS) with genital ambiguity. The testes differentiate normally. In newborns with CAIS, AMH is not as high as expected, probably because the levels of FSH remain low. In newborns with PAIS gonadotropins and AMH are elevated. During childhood AMH remains within normal male range in both conditions. At puberty, in CAIS AMH is abnormally elevated for the high levels of circulating testosterone. In PAIS intratesticular testosterone is capable of causing an incomplete inhibition of $\mathrm{AMH}$, which is nonetheless inadequately high for the levels of circulating testosterone (1). The persistant Müllerian duct syndrome (PMDS) occurs due to mutations in the genes encoding AMH or its receptor (AMH receptor type II). In this condition boys have normal male external genitalia but with persistent internal Müllerian structures. Patients with PMDS could be AMH-negative, with undetectable AMH levels indicative of AMH gene defects or AMH-positive due to mutations in the AMH receptor gene or, in exceptional cases, mutations affecting AMH bioactivity $(6,8)$.

\section{Klinefelter syndrome (47, XXY)}

Klinefelter syndrome and its variants (48, $\mathrm{XXXY}$, etc.) is characterized by tall stature, gynecomastia, small testes and hypergonadotropic hypogonadism in late puberty and adulthood. The levels of $\mathrm{AMH}$ before puberty are normal but after puberty fall to subnormal values because of the progressive testicular degeneration. Thus AMH is a useful marker for the testicular function in these patients (6).

\section{Hypogonadotropic hypogonadism}

In infants with hypogonadotropic hypogonadism AMH is low, while in boys with untreated central hypogonadism at pubertal age serum AMH is elevated for age, because of insufficient down regulation by 
Anti-Müllerian hormone - a hormone with narrow spectrum of action and broad spectrum of clinical significance

testosterone, but lower for the patient's Tanner stage, because of lack of FSH stimulus (1).

\section{Central precocious puberty}

Grinspon et al. found that in 6 untreated boys with central precocious puberty diagnosed at a median age of 3 years the AMH levels were low for their chronological age because the inhibitory effect of androgens on $\mathrm{AMH}$ secretion dominated over the stimulatory effect of FSH. During treatment with $\mathrm{GnRH}$ agonists AMH attained normal prepubertal values. The authors conclude that this could make AMH a useful marker for monitoring the efficacy of the treatment (9).

\section{$A M H$ - clinical use in the female}

In the female $\mathrm{AMH}$ is a clinically useful and more accurate than FSH marker for ovarian reserve. It is used for improvement of infertility treatment, planning of pregnancies, menopause prediction, monitoring of ovarian damage by medications and procedures, detection of PCOS (polycystic ovary syndrome) and POI (premature ovarian insufficiency) (4). Recombinant AMH is a promising therapeutic agent for treatment of gynecologic tumors and endometriosis $(10,11)$.

\section{Young cancer survivors}

In the last decades combined chemo- and radiotherapy for malignancies in girls and young females has led to an improved survival rate and quality of life after anticancer treatment, including ovarian function and reproductive capacity, has become an important issue $(12,13)$. Total body irradiation (TBI) or irradiation of the pelvic region and chemotherapy regiments including alkylating agents cause damage to ovarian follicles mainly by increased apoptosis. This damage can be permanent, leading to POI and infertility or in other cases, the ovarian function may recover. Follicular depletion may occur even if regular menstrual cycle has recovered (12). The Childhood Cancer Survivor Study (CCSS) has shown that premature menopause occurs in $8 \%$ of the cancer survivors and depends on age, dose of irradiation to the ovaries and the cumulative dose of the alkylating agents (14). With higher risk of impaired fertility, more than $80 \%$ are patients after TBI, megachemotherapy, irradiation for pelvic tumors, metatstatic soft tissue sarcomas and Hodgkin lymphomas treated with alkylating agents, independently of age at treatment (pre- or postpubertal). In these patients progressive lowering of AMH during treatment is observed without recovery after treatment, suggesting a profound loss of the primordial follicle pool (13, 15). Thus AMH is an early sensitive marker of reduced ovarian reserve in young cancer survivors.

\section{Hyperandrogenism}

Polycystyc ovary syndrome (PCOS) affects approximately $6 \%$ of the women in reproductive age and results in menstrual disorders and infertility. The diagnostic criteria for PCOS are based on the 2003 Rotterdam consensus and include: oligo- and/ or unovulation, clinical or biochemical hyperandrogenism and ultrasound findings of more than 12 follicles with diameter of 2-9 $\mathrm{mm}$, or ovarian volume of more than $10 \mathrm{~cm}^{3}$ (16). Levels of AMH in PCOS are 2-3 times higher than in normally ovulating and menstruating women and are positively correlated to the androgen level. They begin to decline 5 years later than in healthy women. Because AMH is not affected by the menstrual cycle or use of oral contraceptives it can be used as a biological marker for PCOS and the degree of impairment of folliculogenesis $(17,16)$. In a study of 71 women with PCOS and 71 controls at a reproductive age between 18 and 35 years Wiweko et al. found that with a cutoff value of $4.45 \mathrm{ng} / \mathrm{ml}$, the serum AMH levels had a sensitivity of $76.1 \%$ and a specificity of $74.6 \%$ to predict PCOS. The mean levels of AMH were highest in the phenotypes of unovulation, hyperandrogenism and polycystic ovaries (16). Lin et al. obtained similar sensitivity and specificity values, $70 \%$ and $76 \%$ respectively, but at higher cutoff value of AMH $-7.3 \mathrm{ng} / \mathrm{ml}$ (18). Pawelczak et al. studied 23 adolescent females with PCOS (mean age 15.2 \pm 1.8 years) and 12 age and BMI matched controls (mean age 14.1 \pm 1.7 years) and found significantly higher AMH levels in the PCOS group $(6.78 \pm 3.55 \mathrm{ng} / \mathrm{ml}$ vs $3.38 \pm 1.48 \mathrm{ng} / \mathrm{ml})$. The authors found also a positive correlation between AMH levels and ultrasound measured ovarian volume and concluded that AMH could supplement ultrasound in the clinical diagnosis and follow-up of PCOS (19).

Granulosa cell tumors represent 2-5\% of ovarian neoplasms and in prepubertal girls they may present as precocious puberty. AMH is a reliable and specific marker of granulosa cells and their activity and is in the male concentrations range in such girls. Be- 
cause larger tumors gradually lose their AMH expression, there is an inverse correlation between the tumor size and the AMH level (6). Changes in AMH levels are also a marker for success of the treatment or recurrence of the tumor (10).

\section{Diminished ovarian reserve}

In women with ovarian insufficiency serum AMH level is low and is the earliest and most accurate serum marker for decline in ovarian reserve. Thus AMH may be the earliest clinical means of detecting women at risk to develop POI or premature ovarian failure (POF) (4).

\section{In-vitro fertilization (IVF)}

Serum AMH concentration together with antral follicular count (AFC) are among the best markers for predicting ovarian response to gonadotropin stimulation in IVF treatment. However, the value of $\mathrm{AMH}$ as a predicting tool for live-birth rate and oocyte quality remains controversial $(20,4)$.

\section{Gynecologic tumors and endometriosis}

It was first suggested by Professor Robert Scully, an expert in ovarian cancer from the Pathology Department of the Massachusetts General Hospital, that the ability of AMH to induce apoptosis and to inhibit cell division in a highly specific manner can be used for treatment of cancers of Müllerian duct origin. He proposed such treatment for epithelial ovarian cancers, which recapitulate embryonic Müllerian histology (after 10). More recent experiments found that cervical and endometrial cancers, as well as some non-Müllerian cancers of the breast, prostate and ocular melanoma, could be a potential target for treatment with recombinant AMH because these tissues express AMH type II receptors. Scientists propose initiation of phase I trials as soon as sufficient clinical material is available (10). In endometriosis there is implantation of endometrial glands and stroma outside the uterine cavity, most commonly on the visceral and peritoneal surfaces, but also, though rarely, in the pleura, pericardium or brain. The prevalence of this condition in the general female population is around $6-10 \%$. It is usually associated with infertility, pelvic pain, dispareunia and though quite common, it is frequently misdiagnosed. The current treatment modalities, which include oral contraceptive pills, Danazol, gonadotropin releasing hormone analogues, progestins, androgens and surgical inter- ventions have high recurrence rates of up to $45 \%$ (21). Recent studies have found that AMH type II receptors are expressed in the endometrium in the adult female where probably AMH in a paracrine fashion negatively regulates cellular viability (11). In experimental in vitro models of endometriosis Signorile et al. found that AMH was able to inhibit cell proliferation and induce apoptosis in endometriosis cells. This data suggests a possible use of AMH as treatment for endometriosis but further studies are needed (11).

\section{CONCLUSIONS}

$\mathrm{AMH}$ is a useful biomarker with important diagnostic and predictive value, both in the male and the female. Its use in the clinical practice is constantly widening. AMH is also emerging as a promising therapeutic agent for treatment of cancers and endometriosis.

\section{REFERENCES}

1. Josso N, Rey RA, Picard JY. Anti-müllerian hormone: a valuable addition to the toolbox of the pediatric endocrinologist. Int J Endocrinol. 2013;2013:674105.

2. Grinspon RP, Rey RA. Anti-müllerian hormone and sertoli cell function in paediatric malehypogonadism. Horm Res Paediatr. 2010;73(2):81-92.

3. Lashen H, Dunger DB, Ness A, Onq KK. Peripubertal changes in circulating antimüllerian hormone levels in girls. Fertil Steril. 2013 Jun;99(7):2071-5.

4. Leader B, Baker VL. Maximizing the clinical utility of antimüllerian hormone testing in women's health. Curr Opin Obstet Gynecol 2014, 26(4):226-36.

5. Lie Fong S, Visser JA, Welt CK, de Rijke YB, Eijkemans MJ, Broekmans FJ, et al. Serum antimüllerian hormone levels in healthy females: a nomogram ranging from infancy to adulthood. J Clin Endocrinol Metab. 2012; 97(12): 4650-5.

6. Lindhardt Johansen $M$, Hagen $C P$, Johansen TH, Main KM, Picard JY, Jorgensen A, et al. Anti-müllerian hormone and its clinical use in pediatrics with special emphasis on disorders of sex development. Int J Endocrinol. 2013;2013:198698.

7. Hughes IA, Houk C, Ahmed SF; LWPES Consensus Group; ESPE Consensus Group. 
Anti-Müllerian hormone - a hormone with narrow spectrum of action and broad spectrum of clinical significance

Consensus statement on management of intersex disorders. Arch Dis Child. 2006;91(7):554-63

8. Ahmed SF, Rodie M. Investigation and initial management of ambiguous genitalia. Best Pract Res Clin Endocrinol Metab. 2010;24(2):197-218

9. Grinspon RP, Andreone L, Bedecarras P, Ropelato MG, Rey RA, Campo SM, et al. Male central precocious puberty: serum profile of anti-müllerian hormone and inhibin B before, during, and after treatment with GnRH Analogue. Int J Endocrinol. 2013;2013:823064

10. Kim JH, MacLaughlin DT, Donahoe PK. Müllerian inhibiting substance/anti-müllerian hormone: a novel treatment for gynecologic tumors. Obstet Gynecol Sci 2014;57(5):343-57

11. Signorile PG, Petraglija F, Baldi A. Anti-mullerian hormone is expressed by endometriosis tissues and induces cell cycle arrest and apoptosis in endometriosis cells. J Exp Clin Cancer Res. 2014 May 29;33:46.

12. Peigne $M$, Decanter C. Serum AMH level as a marker of acute and long-term effects of chemotherapy on the ovarian follicular content: a systematic review. Reprod Biol Endocrinol. 2014 Mar 26;12:26

13. Krawzcuk-Rybak M, Leszczynska E, Poznanska M, Zelazowska-Rutkowska B, Wysocka J. Antimüllerian hormone as a sensitive marker of ovarian function in young cancer survivors. Int J Endocrinol. 2013;2013:125080

14. Green DM, Sklar CA, Boice JD Jr, Mulvihill JJ, Whitton JA, Stovall M, et al. Ovarian failure and reproductive outcomes after childhood cancer treatment: results from the childhood cancer survivor study. J Clin Oncol. 2009 May 10;27(14):2374-81

15. Brougham M F, Crofton PM, Johnson EJ, Evans N, Anderson RA, Wallace WH. Anti-müllerian hormone is a marker of gonadotoxicity in preand postpubertal girls treated for cancer: a prospective study. J Clin Endocrinol Metab. 2012 Jun;97(6):2059-67

16. Wiweko B, Maidarti M, Priangga MD, Shafira N, Fernando D, Sumapraja K, et al. Anti-mullerian hormone as a diagnostic and prognostic tool for PCOS patients. J Assist Reprod Genet. 2014 Oct;31(10):1311-6

17. Bungum L, Franssohn F, Bungum M, Humaidan $\mathrm{P}$, Giwercman A. The circadian variation in anti- müllerian hormone in patients with polycystic ovary syndrome differs significantly from normally ovulating women. PLoS One. 2013 Sep 4;8(9):e68223

18. Lin YH, Chiu WC, Wu CH, Tzeng CR, Sen Hsu C, Hsu MI. Antimullerian hormone and polycystic ovary syndrome. Fertil Steril. 2011;96(1):230-5.

19. Pawelczak M, Kenigsberg L, Milla S, Liu YH, Shah B. Elevated serum anti-müllerian hormone in adolescents with polycystic ovary syndrome: relationship to ultrasound features. J Pediatr Endocrinol Metab. 2012; 25(9-10): 983-9.

20. Li HW, Lee VC, Lau EY, Yeung WS, Ho PC, Nq $\mathrm{EH}$. Ovarian response and cumulative live birth rate of women undergoing in-vitro fertilisation who had discordant anti-müllerian hormone and antral follicle count measurements: a retrospective study. PLoS One. 2014 Oct 14;9(10):e108493

21. Giudice LC, Kao LC. Endometriosis. Lancet 2004 Nov 13-19;364(9447):1789-99. 